\title{
A Study on the Effect of Neurogenesis and Regulation of GSK3 $\beta /$ PP2A Expression in Acupuncture Treatment of Neural Functional Damage Caused by Focal Ischemia in MCAO Rats
}

\author{
Ding Luo, ${ }^{1}$ Xiaonong Fan, ${ }^{2,3,4}$ Congcong Ma, ${ }^{1}$ Tongtao Fan, ${ }^{1}$ \\ Xiaoguang Wang, ${ }^{1}$ Nvzi Chang, ${ }^{1}$ Lingxin Li, ${ }^{1,5}$ Yanan Zhang, ${ }^{2,3,4}$ \\ Zhihong Meng, ${ }^{4,6}$ Shu Wang, ${ }^{2,4}$ and Xuemin Shi ${ }^{2,4,6}$ \\ ${ }^{1}$ Postgraduate Department of Tianjin University of Traditional Chinese Medicine, Tianjin 300193, China \\ ${ }^{2}$ The Acupuncture \& Moxibustion Institute, First Teaching Hospital of Tianjin University of Traditional Chinese Medicine, \\ Tianjin 300193, China \\ ${ }^{3}$ The Third-Level Acupuncture Dose-Effect Laboratory of the State Administration of Traditional Chinese Medicine, \\ Tianjin 300193, China \\ ${ }^{4}$ Tianjin Key Laboratory of Acupuncture \& Moxibustion Science, Tianjin 300193, China \\ ${ }^{5}$ Department of Rehabilitation Medicine, West China Hospital, Sichuan University, Chengdu, Sichuan 610041, China \\ ${ }^{6}$ Department of Acupuncture and Moxibustion, First Teaching Hospital of Tianjin University of Traditional Chinese Medicine, \\ Tianjin 300193, China
}

Correspondence should be addressed to Xuemin Shi; tjdrshi@msn.com

Received 27 March 2014; Revised 25 May 2014; Accepted 9 June 2014; Published 10 July 2014

Academic Editor: Yi-Hung Chen

Copyright (c) 2014 Ding Luo et al. This is an open access article distributed under the Creative Commons Attribution License, which permits unrestricted use, distribution, and reproduction in any medium, provided the original work is properly cited.

$170 \mathrm{SD}$ rats were randomly divided to five groups. Rats in model group, no-acupuncture group, and acupuncture group were subjected to MCAO surgery. Acupuncture group received 3 consecutive acupuncture treatments at a parameter that deep in $2 \mathrm{~mm}$ towards apex nasi and thrust/lifted at 3 times per second for 1 minute, while model group and no-acupuncture group were no-intervention control groups. Serious neural functional damage and sharp decrease of cerebral blood flow, obvious infarction volume, increased nestin mRNA expression, and immunopositive cells population (nestin ${ }^{+}, \mathrm{BrdU}^{+}$and nestin/BrdU ${ }^{+}$) were found in MCAO rats which had not been observed in normal group and sham-operated group. However, the damage was attenuated by rat's "self-healing" capacity 3 days after MCAO. And the "self-healing" capacity can be strengthen by acupuncture treatment through increasing cerebral blood flow, neurogenesis, and regulation of gene transcription or GSK-3 $\beta$ and PP2A expression. In conclusion, the present study indicates that the underlying mechanism of acupuncture treatment on neural functional damage caused by focal ischemia injury is a multiple interaction which may involve improved cerebral blood supply, neurogenesis, and regulation of gene transcription or GSK-3 $\beta$ and PP2A expression in MCAO rats.

\section{Introduction}

Stroke is the leading cause of death and disability and thus a tough challenge for healthcare system and a heavy social burden in China [1]. It is characteristic of ischemia accounting for $43 \%$ to $79 \%$ of stroke and a belt of high incidence existed in many provinces [2]. Limited by the short time window for thrombolytic therapy, high hopes have been placed on neuron plasticity for treating stroke caused by ischemia. As evidence supplied by animal researches, endogenous neurogenesis occurs in subventricular zone (SVZ) [3] and dentate gyrus of subgranular zone (SGZ) in hippocampus [4-6] throughout mammalian life, which can be triggered by many factors such as ischemic insult [7], traumatic injury [8], apoptosis [9], VEGA [10], and inflammation [11]. These neural stem cells are capable of self-renewal and differentiation that may compensate the damaged neurons and neurogliocytes for subsequent functional recovery, which can be marked by special cellular 


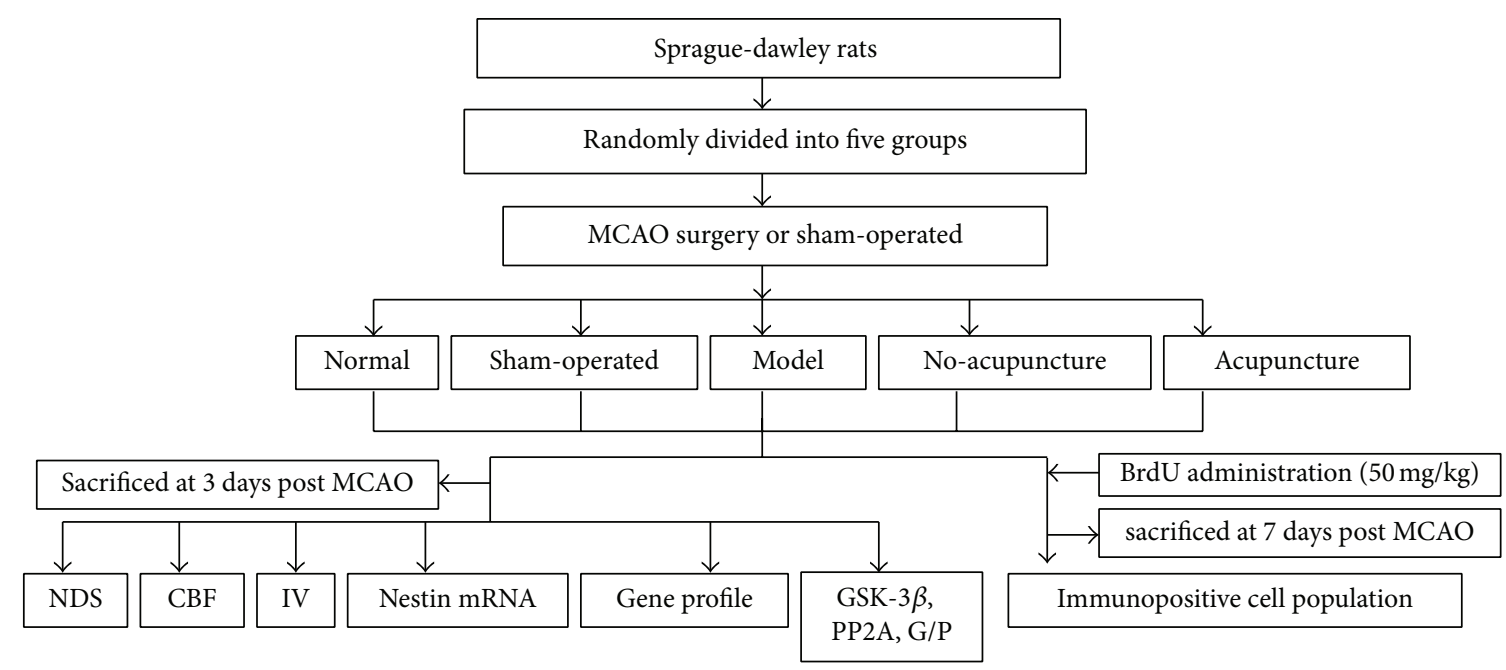

FIGURE 1: Schematic diagram for methodologies: a total of 170 adult male SD rats were randomly divided into normal, sham-operated, model, no-acupuncture, and acupuncture group. Rats in model group euthanatized immediately after MCAO while other groups at 3 days for subsequent investigation of neural deficit score (NDS), cerebral blood flow (CBF), nestin mRNA expression, microarray analysis, and enzyme-linked immunoabsorbent assay. Another sets of rats sacrificed 7 days for immunopositive cell counting.

marker protein such as nestin. Thus, treatment which can amplify endogenous neurogenesis should have great potential for ischemic stroke treatment.

Acupuncture is a traditional therapy derived from ancient China, well-known by its good therapeutic effects on many diseases with long period of large body clinic practice. According to a randomized controlled prospective clinic trial conducted by our team [12], acupuncture effectively improves the self-care ability and life quality of patients with onset of ischemic stroke. Coincidences with the result of clinic trial, animal researches also demonstrate a neuroprotective effect on MCAO rats treated by acupuncture, which is relative to increased brain blood flow, reduced ischemic infarction volume, and lowered neuronal cell death rate in acupuncture group under the proper stimulated parameter on special acupoint $[13,14]$.

However, the mechanism underlying about how acupuncture works is still unknown. We, therefore, try to reveal the mechanism from accessing neural function deficit, infarction volume, brain blood flow, neural stem cell population, and marker gene expression by neural deficit score, 2,3,5triphenyl tetrazolium chloride staining, laser-Doppler flow meter, immunofluorenscence double-staining, and real-time polymerase chain reaction. In addition, we also utilize microarray to identify gene profile of acupuncture treatment and enzyme-linked immunoabsorbent assay (ELISA) to verify microarray results. The study aims at elucidating the exact mechanism of acupuncture treatment on ischemia stroke.

\section{Materials and Methods}

2.1. Animals and Groups. A total of 170 adult male SpragueDawley rats which were purchased from Experimental Animal Center of Academy of Military Medical Sciences weighting from 250 to $280 \mathrm{~g}$ were employed. All animals were housed in a conditioned environment (12 hour-light/12 hourdark circle, humidity $55 \pm 5 \%$, and free access to food and water). All the experimental protocols were approved by local animal ethical committee and consistent with experimental animal use guidelines. Rats were randomly divided into five groups as normal group, sham-operated group, model group, no-acupuncture group, and acupuncture group. Rats in model group, no-acupuncture group, and acupuncture group subjected to MCAO surgery. Acupuncture group received 3 consecutive acupuncture treatments. The model group and no-acupuncture group were no-intervention control groups for acupuncture group. Rats in model group decapitated immediately after behavior measurement by neural deficit scores when recovering consciousness from MCAO surgery. But rats in no-acupuncture group were killed at the same time point as acupuncture group and the difference between two groups was received acupuncture treatment or not (one set sacrificed at 3 days after MCAO for CBF, IV, PCR, and gene microarray measurement while the other set killed at 7 days for immunopositive population counting). It was meant to investigate the immediate damage caused by ischemia and the "self-repairing" ability of MCAO rats. The experimental protocols were shown in Figure 1.

2.2. Permanent Middle Cerebral Artery Occlusion. Permanent middle cerebral artery occlusion was performed as previously described $[13,15]$. In brief, rats were anesthetized with $10 \%$ chloral hydrate $(250 \mathrm{mg} / \mathrm{kg})$ by intraperitoneal injection. A 2 to $2.5 \mathrm{~cm}$ incision was cut in the centre of neck. Left common carotid artery (CCA), external carotid artery (ECA), and internal carotid artery (ICA) were exposed and isolated. After CCA was clamped and ECA was ligatured by $0^{\#}$ suture, a nylon filament (diameter $0.265 \mathrm{~mm}$ ) with round tip made by heating near a flame was inserted intraluminally into the ICA about 18 to $20 \mathrm{~mm}$ until a slight resistance was felt (which 
means the tip of the filament reached the origin of middle cerebral artery) and ligatured. Then, we released the clamp in CCA and sutured the incision. Gentamicin was used for anti-infection in incision after surgery. Sham-operated group received the same surgery but without nylon filament insertion. The rats' rectum temperatures were maintained at $37 \pm$ $1^{\circ} \mathrm{C}$ during the surgery by an electric blanket.

2.3. $5^{\prime}$-Bromo-2'-deoxyuridine (BrdU) Administration and Tissue Preparation. BrdU, a thymidine analog that can be integrated into DNA synthesis during S-phase of the cell circle, was employed for capturing the proliferated cell population after ischemic infarction. BrdU (Sigma, USA) was dissolved in $40-50^{\circ} \mathrm{C}$ warm saline $(0.9 \% \mathrm{NaCl}$ in sterile $\mathrm{H}_{2} \mathrm{O}$ ). Rats from each group received fresh dissolved $\mathrm{BrdU}$ solution injection intraperitoneally at a dose of $50 \mathrm{mg} / \mathrm{kg}$ [16] for 7 consecutive days twice daily 2 hours after the onset of the permanent middle cerebral artery occlusion. But rats in model group only received one BrdU injection before euthanasia. Then rats were euthanasized under over anesthesia, followed by transcardially perfusing with saline and $4 \%$ paraformaldehyde in $0.1 \mathrm{M}$ phosphate-buffer at $4^{\circ} \mathrm{C}$. Then brains were quickly removed and post-fixed in $12 \%$ formalin solution for 24 hours at room temperature. A $3 \mathrm{~mm}$ coronal block was cut at $3 \mathrm{~mm}$ posterior of optic chiasma and embedded in paraffin, then sectioned at $4 \mu \mathrm{m}$ each. Sections were dried off at $60^{\circ} \mathrm{C}$ after immersing in polylysine and prepared for subsequent examination of immunofluorescence doublestaining.

2.4. Acupuncture Stimulation. Rats in acupuncture group received acupuncture stimulation in consciousness once daily for 3 consecutive days immediately after MCAO performed by a skilled acupuncture practitioner. The DU26 (Shuigou acupoint) located at the junction of the upper one-third and lower two-thirds of the cleft lip midline beneath the nasal septum as described in our previous paper [13] was stimulated by a stainless needle of $0.25 \mathrm{~mm}$ in diameter and $30 \mathrm{~mm}$ in length (Hua Tuo Medical Instruments Co. Ltd Suzhou, China). The needle was inserted deeply in $2 \mathrm{~mm}$ manually towards apex nasi and thrust/lifted stimulated at a parameter of 3 times per second for 1 minute.

2.5. Behavioral Measurements. The behavior of rats at baseline and before euthanasia was assessed by Zausinger' 6-point scale neural deficit scores [17] as previously reported which was performed by a single researcher who was blind to the experimental groups. The scale was carried out as follows: (0), without spontaneous activity; (1), falling to the contralateral side; (2), severe circling when tail pull; (3), lowered resistant to contralateral push; (4), unable to extend the contralateral forelimb; (5), no deficit. The lower the score is, the worse the neural functional lesion is.

2.6. Infarction Volume Assessment. 2,3,5-Triphenyl tetrazolium chloride (TTC) staining was adopted to quantify the infarction volume as previously reported [13]. Briefly, brains were quickly removed after decapitation and then frozen in $-20^{\circ} \mathrm{C}$ for $30 \mathrm{~min}$. The frozen brains were sectioned equally into 5 pieces (about $2 \mathrm{~mm}$ each). All sections were stained with $0.4 \%$ TTC (Sigma, USA) solution for $20 \mathrm{~min}$ at $37^{\circ} \mathrm{C}$ followed by fixation in $12 \%$ formalin solution for $2 \mathrm{~min}$. Then the TTC stained slices were photoed by Olympus fe- 240 digital camera (Pooher Photoelectric Technology Co., Ltd., Shanghai, China) and analyzed by Image Analysis Software (Image-pro Plus 6.0, Media Cybernetics, Inc., Bethesda, MD, USA). The infarction volume was presented as a percentage of the total ipsilateral hemispheric volume which can be calculated by following equation: [(contralateral hemispheric volume - ipsilateral hemispheric volume)/contralateral hemispheric volume $] \times 100 \%[18,19]$.

2.7. Cerebral Blood Flow Observation. A laser-Doppler flow meter was employed for observing cerebral blood flow in cerebral pia mater by using a flexible fibre optic to monitor moving red blood cells. In brief, rat's head was secured in a stereotactic frame under anesthesia by $10 \%$ chloral hydrate $(250 \mathrm{mg} / \mathrm{kg})$ intraperitoneal injection. A center incision was made to expose the skull. A $1.0 \mathrm{~mm} \times 2.00 \mathrm{~mm}$ hole ahead of the bregma was carefully drilled by a dental drill while superfused by warm saline. Then the cerebral blood flow was measured by a laser-Doppler flow meter (Moor-DRT4, Wilmington, DE, USA). The laser-Doppler probe recorded the cerebral blood flow on intact dura mater for 1 minute. Data was analyzed by bundled software.

2.8. Immunofluorescence Double-Staining. Immunofluorescence double-staining was employed to inspect the endogenous neurogenesis with antibodies against BrdU and nestin. Paraffin-embedded sections were dewaxed in dimethylbenzene and hydrated in gradient ethanol after heating at $70^{\circ} \mathrm{C}$ for 2 hours, followed by antigen retrieval in citrate buffer $(\mathrm{pH}=$ 6.0) in microvan at medium heating for $10 \mathrm{~min}$ and cooled in room temperature for 1 hour. Slices were then washed in $0.01 \mathrm{M}$ PBS 3 times for 5 minutes each time, subsequently despiralized in $2 \mathrm{~N} \mathrm{HCl}(1: 5)$ for 30 minutes at $37^{\circ} \mathrm{C}$ and blocked in $10 \%$ goat serum for 30 minutes. The blocked slices were then incubated in BrdU and nestin combination primary antibodies which dissolved in $0.01 \mathrm{M}$ PBS with the dilution data $1: 50$ and $1: 100$, respectively, overnight at $4^{\circ} \mathrm{C}$. And the secondary antibody Goat anti-Mouse AlexaFluor 488 $(1: 300)$ and Goat anti-rabbit AlexaFluor 594 (1:300) were used on the second day. Negative controls received the same treatment omitting the primary antibodies and showed no specific staining. 5 visual regions in penumbra were selected for immune positive cells counting under a 400x microscope.

2.9. Real-Time Polymerase Chain Reaction. The cortex, hippocampus, and striatum of rats brain were rapidly dissected and total RNA was purified by using Trizol Reagent (Invitrogen, USA) according to the manufacturer's instructions. The complementary DNA was synthesized by Shanghai Science \& Technical Co. (Shanghai, China). Forward and reverse primers were $5^{\prime}$-CTCTTGGCTTTCTGGACCCC- ${ }^{\prime}$ and $5^{\prime}$-CACAGGAGTCTCAAGGGTATTAGGC- $3^{\prime}$ for nestin, $5^{\prime}$-CAGCCTTCCTTCCTGGGTATG- $3^{\prime}$ and $5^{\prime}$-TAGAGCCACCAA- 
TCCACACAG- ${ }^{\prime}$ for actin. All samples were normalized by actin. Each sample was tested in triplicate. Relative gene expression was measured as $2^{-\Delta \Delta C T}$ method [20].

2.10. Microarray Analysis. To observe the effects of acupuncture treatment on gene transcription after ischemia, microarray analysis was employed. Ipsilateral brains were collected and stored in liquid nitrogen preparing for microarray analysis. Total RNA was extracted using a Trizol reagent (Invitrogen, USA) according to the manufacturer's instructions and quantified by ultraviolet spectrophotometer and polyacrylamide gel electrophoresis. Purified RNA was converted to cDNA and amplified by using Illumina TotalPrePRNA kit (Illumina, USA). Followed sample labeling, hybridization (Gene Expression Hybridization Kit Agilent p/n 5188-5242), feature extraction (Agilent G4450AA Feature Extraction software 10.7), and image scanning (Agilent Scan Control software) were utilized Agilent standard protocol. Briefly, degenerated hybridization solution was sampled on slides followed by hybridized in hybridization chamber for 1 2 hours at $42^{\circ} \mathrm{C}$ as prehybridization. Mixture combined reverse transcription product and hybridization solution was degenerated in $95^{\circ} \mathrm{C}$ for 2 minutes while prehybridized slides degenerated in $95^{\circ} \mathrm{C}$ for 30 seconds. Then, target DNA in chips was hybridized at $42^{\circ} \mathrm{C}$ overnight. After hybridization, the arrays were washed twice for 10 minutes each in washing solution. Fluorescence signals from each microarray were collected by DNA microarray scanner and converted to original data. The data was preprocessed by subtract in limma package software. Probe expression in chips was represented as mean. Gene expression values between groups that increased by 2 -fold or decreased by 0.5 -fold were considered to be significant difference $(P<0.005)$. Gene Ontology $(G O)$ analysis was employed to analyze functional enrichment. Pathway enrichment analysis was utilized to analyze involved pathway by searching in KEGG (Kyoto encyclopedia of Genes and Genomes) data base. Expression profile chip in each group had 5 times biological repeat.

2.11. Enzyme-Linked Immunoabsorbent Assay (ELISA). The expression of GSK- $3 \beta$ and PP2A was evaluated by enzymelinked immunoabsorbent assay. Ipsilateral brains from each group were divided into cortex, hippocampus, and striatum after decapitation. The tissue was homogenized and purified by centrifugation. GSK- $3 \beta$ and PP2A Immunoassay ELISA kit (R\&D, USA) were used to determine GSK- $3 \beta$ and PP2A level in supernatants according to the manufacturer's instructions. Absorbance of each sample was measured using a microplate reader at a wavelength of $450 \mathrm{~nm}$. All the samples were measured in duplicate.

2.12. Statistical Analysis. All data were analyzed by SPSS 17.0 software and present as Mean \pm SD except for NDS and microarray. One-way ANOVA followed by LSD and a posthoc Mann-Whitney $U$ test was used for analyzing the data. The standard statistical function of R/bioconductor, $t$-test, Fcriterion of ANOVA, and FDR was performed for determined

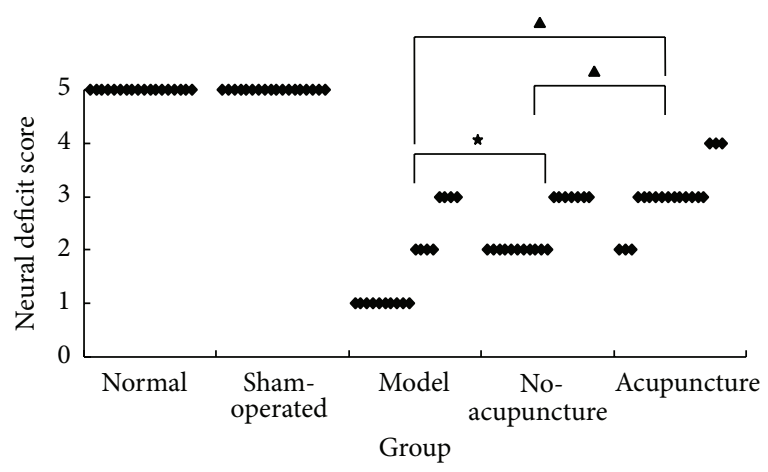

FIgURE 2: Acupuncture attenuated neural functional deficit. Neural deficit score (NDS) was employed for evaluating the neural functional deficit $(n=18)$. The lower the score is, the worse the damage is. There were significant differences among MCAO groups: noacupuncture group got higher scores than model group $(\star P<0.05)$ suggesting a "self-repairing" ability of MCAO rats. Acupuncture group got the highest scores in MCAO group while lower scores in model and no-acupuncture group $(\mathbf{\Delta} P<0.05)$ which meant that acupuncture attenuated neural damage after MCAO but did not reach to a normal level.

genes differential expression of microarray analysis. Possibility values of $<0.05$ were considered as statistically significant.

\section{Results}

3.1. Acupuncture Alleviated Neural Functional Deficit. Neural functional deficit was evaluated by Zausinger et al. [17] 6 point scale at baseline and before euthanatized in MCAO groups and the corresponding time point of normal and sham-operated group. Mann-Whitney $U$ test was conducted to analyze the neural deficit score. As shown in Figure 2, there were significant differences among MCAO groups and normal, sham-operate group $(P<0.05)$ which means MCAO caused a dramatic damage on neural function. Compared two no-intervention groups, no-acupuncture group shown an attenuation of neural damage to model group suggesting a self-repairing capacity of rat post ischemia infarction. But acupuncture group shown a distinct increased in NDS compared to other MCAO groups while still lower than normal and sham-operated group indicating that acupuncture relieves the neural functional damage but did not reach to a normal level.

3.2. Acupuncture Improved Cerebral Blood Flow. As shown in Figure 3, cerebral blood flow in rats of model group and no-acupuncture group were sharply decreased after onset of $\mathrm{MCAO}$. In contrast, there was a significant increase of $\mathrm{CBF}$ in acupuncture group compared to model group and no-acupuncture group, suggesting the shortage of brain blood supply caused by middle cerebral artery occlusion could be rectified by acupuncture which approximately reached to the normal level.

3.3. Acupuncture Did Not Decrease the Infarction Volume. To observe neural protective effect of acupuncture on MCAO 


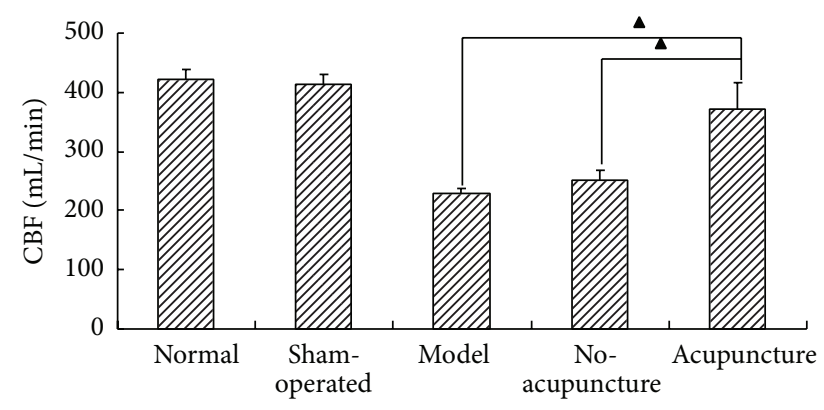

FIGURE 3: Acupuncture improved cerebral blood flow (CBF). A sharp decrease in cerebral blood flow was found in model group and no-acupuncture group compared to normal and sham-operated group. No significant difference was found between model group and no-acupuncture group. But acupuncture increased the cerebral blood flow induced by MCAO $(\boldsymbol{\Delta} P<0.05)(n=6)$.

rats, TTC staining was utilized for evaluating the infarction volume. Conspicuous infarction formed in brain of rats in model group, no-acupuncture group, and acupuncture group (shown in Figure 4(a)), whereas no infarction observed in normal and sham-operated group. No marked difference was found between model group and no-acupuncture group. There was a tendency of decrease in acupuncture group compared to model group and no-acupuncture group but no significant difference has been found.

3.4. Acupuncture Enhanced the Neurogenesis in MCAO. Immunofluorescence double-staining combined with realtime polymerase chain were adopted for examining the endogenous neurogenesis. BrdU co-labeled with nestin (a special marker for neural stem cells in central nervous system (CNS)) were employed for labeling neural stem cells [21]. Furthermore, RT-PCR was used to quantify the mRNA expression of nestin in cortex, hippocampus, and striatum, respectively.

By immunofluorescence double-staining, a large number of $\mathrm{BrdU}^{+}$, nestin ${ }^{+}$, and BrdU/nestin ${ }^{+}$cells in MCAO groups were observed as shown in Figure 5, whereas no immunepositive cells were found in normal and sham-operated group. There was also a significant increase of immunopositive cells in acupuncture group versus model group and noacupuncture group (shown in Figures 5(b), 5(c), and 5(d)), suggesting acupuncture treatment induced proliferation of neural stem cells in a certain extent. And BrdU ${ }^{+}$cells obviously increased in no-acupuncture group compared to model group. However, no distinct difference was found in nestin ${ }^{+}$ and BrdU/nestin ${ }^{+}$between no-acupuncture group and model group (shown in Figures 5(c) and 5(d)).

Consistent with the results of immunofluorescence double-staining, model group shown a high expression of nestin mRNA compared to normal and sham-operated group especially in striatum followed by hippocampus and cortex suggesting there was different expression tendency in three cerebral regions after ischemia. However, nestin expression of no-acupuncture group increased in cortex and hippocampus but decreased in striatum (shown in Figure 6). As to model group, no-acupuncture group supposed to be a "self-repair" control group, because neither of them received any intervention but sacrificed at 3 days after the model group. From nestin expression of cortex, hippocampus, and striatum, we found that different cerebral regions shown different "selfrepair" capacity. And the "self-repair" capacity could be enhanced by acupuncture stimulation; as demonstrated in Figure 6, nestin mRNA was significantly increased in cortex while slightly increased in hippocampus and decreased in striatum compared to model group and no-acupuncture group. Cortex may be more sensitive for acupuncture stimulation than hippocampus and striatum.

3.5. Acupuncture Treatment Evoked Expression of Phosphorylation and Cell Proliferation Relative Genes. To obtain the gene expression stimulated by acupuncture treatment after ischemia, we performed a microarray analysis to identify differentially expressed genes. According to analytic strategy mentioned above ( $P$ adjust $\leq 0.01$ ), we first determined 12350 differentially expressed genes among normal, shamoperated, model, no-acupuncture, and acupuncture groups. All significantly and differentially expressed genes were analyzed by Gene Ontology (GO) analysis to identified relative biological process, cellular component, and molecular functions. As shown in Table 1, total of 444 biological processes, 164 molecular functions, and 47 cellular components were involved in the physiopathological processes. And the top 10 enriched GO biological processes were included such as tyrosine phosphorylation, immunologic tolerance, protein negative regulated, and cell proliferation. Additional, KEGG analysis was employed for pathway enrichment. The top 10 enriched pathways were involved in neural signaling transduction, glutamate metabolism, neuroactive ligand-receptor interaction, complement and coagulation cascades, biosynthesis of steroids, and cytokine-cytokine receptor interaction as demonstrated in Table 2. In general, the differentially expressed genes regulated by acupuncture were mainly belonging to biological processes of metabolism, phosphorylation, cell proliferation, and neural signaling transduction.

3.6. Acupuncture Made a Positive Regulation on GSK-3 $\beta$, $P P 2 A$, and $G / P$. Microarray analysis was shown biological processes of metabolism, phosphorylation, and cell proliferation relevant to neural protective effect after ischemia. Thus, to verify the microarray analysis results, we evaluated GSK-3 $\beta$ and PP2A expression (which well-accepted have closely connection of biological processes mentioned above) in different cerebral regions of rats by ELISA. As demonstrated in Figure 7(a), GSK-3 $\beta$ expression robust increased in MCAO groups, whereas PP2A expression conspicuous decreased. And expression of GSK- $3 \beta$ in no-acupuncture was decreased meanwhile increased of PP2A expression compared to model group $(P<0.05)$. However, acupuncture shown a regulation effects on expression tendency of GSK-3 $\beta$ and PP2A: it enhanced the increasing of GSK-3 $\beta$ while attenuated decreasing of PP2A $(P<0.05)$. And the similar tendency of GSK-3 $\beta$ and PP2A expression was found in cortex, hippocampus and striatum. The ratio of GSK-3 $\beta$ and PP2A was also dramatically elevated post-ischemia, but decreased 


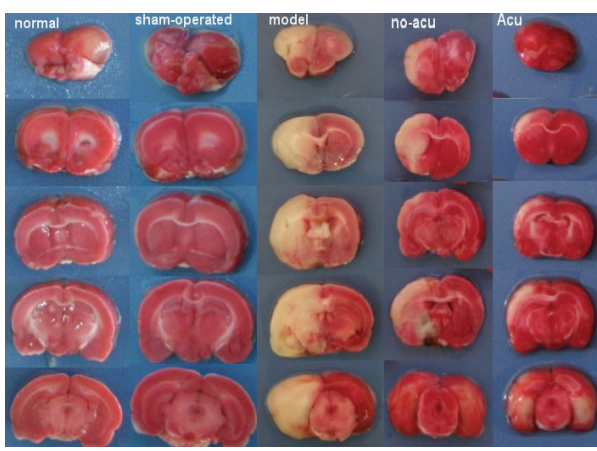

(a)

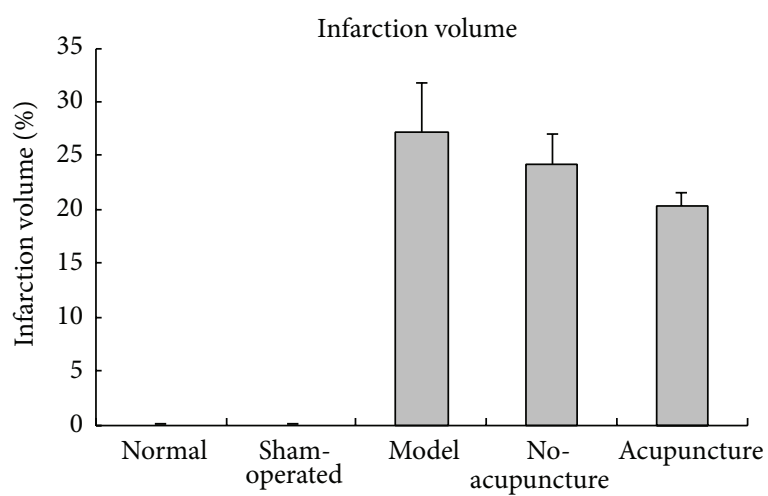

(b)

FIGURE 4: Observation of infarction volume: (a) representative coronal sections stained by TTC from five groups $(n=6)$, (b) quantitative analysis of infarction volume. MCAO caused obvious infarction in MCAO groups compared to normal and sham-operated group. Although acupuncture group shown a tendency of decrease in infarction volume compared to model group and no-acupuncture group, no significant difference has been found.

TABLE 1: The top 10 enriched GO BP of the differentially expressed genes.

\begin{tabular}{|c|c|c|c|}
\hline GO ID & GO term & $P$-value & Gene name \\
\hline GO:0042506 & Tyrosine phosphorylation of Stat 5 protein & 0 & Jak2;Ptk6;Osm;Jak3 \\
\hline GO:0042503 & Tyrosine phosphorylation of Stat 3 protein & 0 & Jak2;Ptk6;Lif;Osm \\
\hline GO:0002513 & Tolerance induction to self-antigen & 0 & Lyn;Foxp3;Tgfb1 \\
\hline GO:0045759 & Negative regulation of action potential & 0 & Chrnb2;Cnr2;Cnr1 \\
\hline GO:2000009 & $\begin{array}{l}\text { Negative regulation of protein localization at cell } \\
\text { surface }\end{array}$ & 0 & Taxlbp3;Gpm6b;Leprot \\
\hline GO:0090191 & $\begin{array}{l}\text { Negative regulation of branching involved in } \\
\text { ureteric bud morphogenesis }\end{array}$ & 0 & Sixl;Tacstd2;Grem1;Bmp4 \\
\hline GO:0090197 & Positive regulation of chemokine secretion & 0 & Il33;C5;Csflr;Il4ra;Chia;Il1rl1 \\
\hline GO:0045356 & $\begin{array}{l}\text { Positive regulation of interferon-alpha } \\
\text { biosynthetic process }\end{array}$ & 0 & Tlr7;Tlr9;Tlr3 \\
\hline GO:0072110 & Glomerular mesangial cell proliferation & 0 & Pdgfb;Pdgfrb;Egr1 \\
\hline GO:0048148 & Behavioral response to cocaine & 0 & Drd2;Drd1a;Abat;Crhr1;Adralb;Snca;Drd3;Drd4;Cdk5 \\
\hline
\end{tabular}

in no-acupuncture group and acupuncture group $(P<0.05)$. The results revealed that it was a temporal profile of GSK$3 \beta$ and PP2A expression, and the ratio of GSK-3 $\beta$ and PP $2 \mathrm{~A}$ $(\mathrm{G} / \mathrm{P})$ positive regulated by acupuncture stimulation.

\section{Discussion}

In the present investigation, we gave experimental evidences that acupuncture treatment enhanced the "self-repairing" capacity of MCAO rats to alleviate neural functional damage induced by ischemia via multiple outcome measurements. Using laser-Doppler flow meter, double-label immunostaining, RT-PCR, microarray and ELISA, we observed that acupuncture obviously increased brain blood flow (Figure 3), neurogenesis (Figure 5) and expression of nestin mRNA (Figure 6), and caused a series changing of genes and GSK$3 \beta / \mathrm{PP} 2 \mathrm{~A}$ (proteins that are relative to biological processes of phosphorylation and cell proliferation) expression (Table 1 and Figure 7).
Although pathological and pathophysiological in brain after ischemia stroke is quite complicated [22], it's ascertaining that endogenous neurogenesis and angiogenesis contribute to neural functional rehabilitation in special area of CNS [23]. As evidences given by recently extensive reports, the beneficial effects of acupuncture on brain ischemic damage in vivo or in vitro mainly focus on microcirculation [24], anti-apoptotic [25], anti-inflammation [26], neuron protection [27], brain metabolism [28]. Our previous studies indicated that acupuncture combined with basic modern medicine treatment showed a great effect on stroke patients' self-care ability and quality of life in clinic trial [12], and the underlying mechanism may be associated with improving cerebral haemodynamics and neuron protective effect under a special stimulated parameter on a special acupionts revealed by animal researches $[13,29,30]$.

In present study, we utilized the optimum parameter $(3$ times per second for 1 minute) as proved by previous study for acupuncture stimulation on DU26 [31]. We found out that cerebral blood flow dramatically increased in acupuncture 


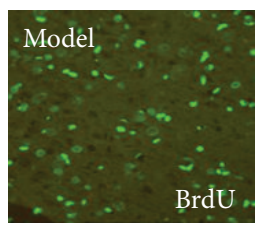

al

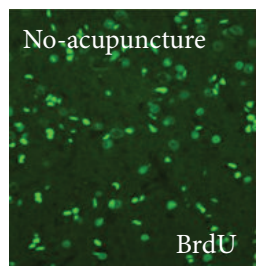

b1

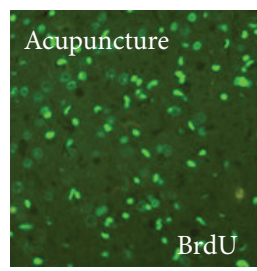

$\mathrm{cl}$

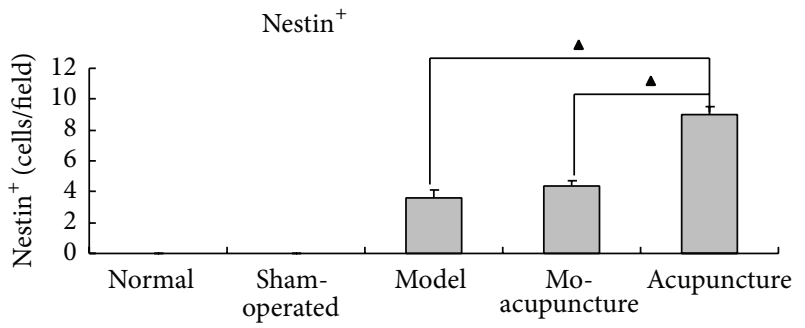

(c)

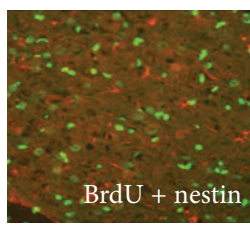

a3

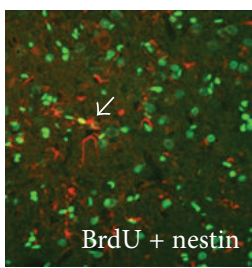

b3

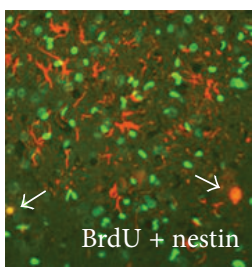

c3

c2

(a)

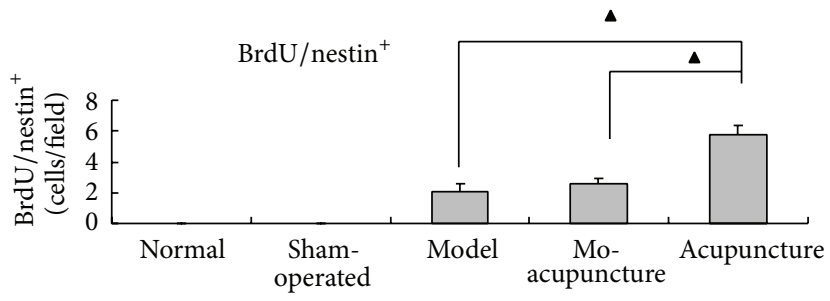

(d)

FIGURE 5: Effects of acupuncture on increasing population of immunopositive cells in penumbra: 5 visual regions in penumbra of each slice were selected for cell counting under a 400x microscope $(n=6)$. (a) Representative images of Immunofluorescence double-staining from model group, no-acupuncture group, and acupuncture group: a1, b1, and c1 labeled for BrdU (green). a2, b2, and c2 labeled for nestin (red). a3, b3, and c3 colabeled for BrdU/nestin. White arrows points to the BrdU/nestin ${ }^{+}$cells. (b), (c), and (d) Quantization of BrdU ${ }^{+}$, nestin ${ }^{+}$, and BrdU/nestin ${ }^{+}$cells: immunopositive cells were found in MCAO groups while not been found in normal and sham-operated group. Acupuncture enhanced $\mathrm{BrdU}^{+}$, nestin ${ }^{+}$, and $\mathrm{BrdU} /$ nestin ${ }^{+}$labeled cells proliferation $(\boldsymbol{\Lambda} P<0.05$, acupuncture group versus model group and no-acupuncture group). More BrdU ${ }^{+}$labeled cells in no-acupuncture group than model group $(\star P<0.05)$.

group. As we know, cerebral blood supply in correspond area is significantly decreased after middle cerebral artery occluded, followed by vulnerable neurons apoptosis rapidly in such a hypoxic-ischemic environment. However, acupuncture increasing the cerebral blood supply in ischemia region is helpful to ameliorate the hypoxic-ischemic situation to a certain extent which may be relevant to regulating vascular caliber, establishing collateral circulation and enhancing angiogenesis [29, 32].

Except for regulating focal cerebral hemodynamics, neurogenesis also has been observed in acupuncture treatment group. BrdU/nestin co-labeled immunofluorescence positive cells and mRNA expression of nestin were detected for evaluating neurogenesis in this study. BrdU is usually used to investigate cell proliferation as it integrates with cell circle during DNA synthesis phase which is considered as a
"Gold standard" to determine neurogenesis [16]. In present research, we have chosen the seventh day after MCAO surgery to observed neurogenesis for a better integration of BrdU into DNA. Nestin is one of intermediate filament proteins that is abundantly and transiently expressed in neural stem cells of developing and developed central neural system and has always been utilized for identifying undifferentiated CNS precursor's proliferation [33-35]. Many BrdU ${ }^{+}$, nestin ${ }^{+}$, BrdU/nestin co-labeled immunofluorescence positive cells were seen in model group in penumbra belt in the study. These endogenous neurogenesis amplified by acupuncture stimulation showed a significant increase of immuno-positive cells in acupuncture group compared with model and noacupuncture group (Figures 5(b), 5(c), and 5(d)). But it is well accepted that generation of neurogenesis only occurs in special regions such as SVZ and SGZ in rodents and humans 


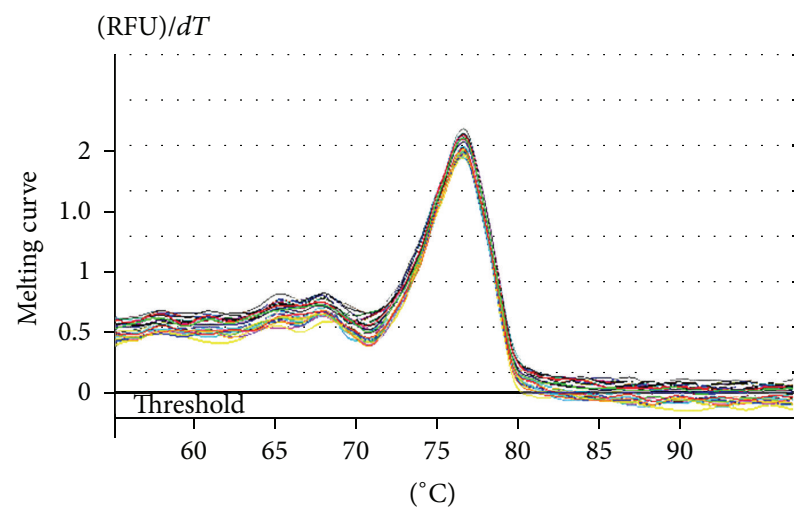

(a)

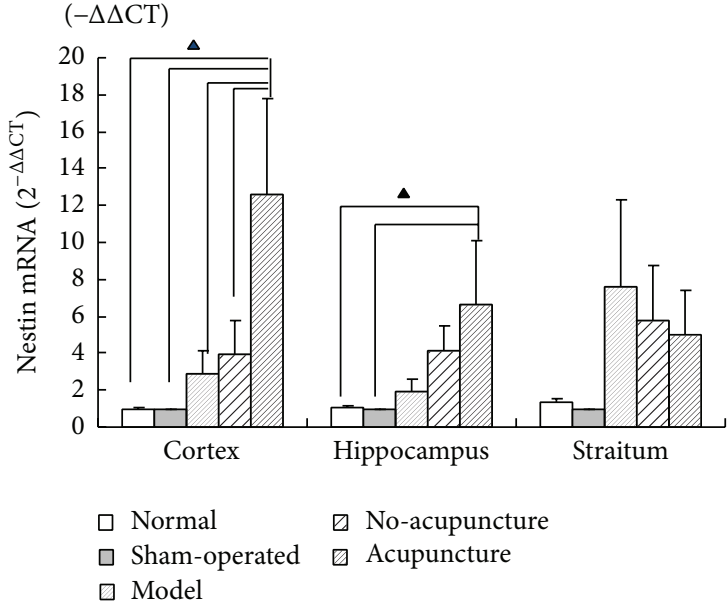

(b)

FIGURE 6: Analysis of the mRNA expression of nestin by real-time PCR: (a) melting curve of nestin by real-time PCR, (b) relative expression of nestin mRNA in cortex, hippocampus, and striatum $\left(2^{-\Delta \Delta C T}\right)$ shows in bar graphs: rare expression of nestin was found in normal and shamoperated group in brain. Nestin mRNA expression of model group greatly increased in striatum and slightly increased in cortex followed by hippocampus. However, expression tendency was different in no-acupuncture group: enhanced in cortex and hippocampus but suppressed in striatum which supposed to be a "self-repairing" control group. Acupuncture group got the highest expression of nestin mRNA in cortex versus other MCAO groups $(\boldsymbol{\Lambda} P<0.05)$ while higher in hippocampus $(\boldsymbol{\Lambda} P<0.05)$ and less in striatum.

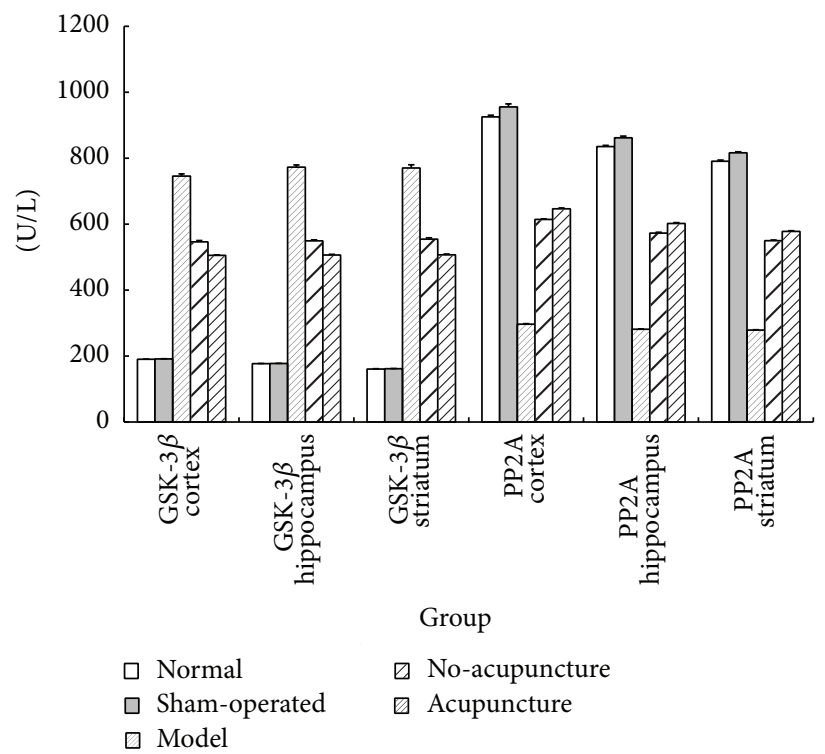

(a)

Sham-operated $\square$ Acupuncture

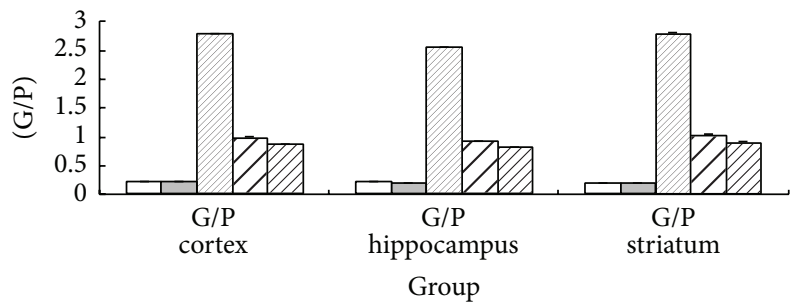

$\square$ Normal $\quad \square$ No-acupuncture

$\square$ Sham-operated $\square$ Acupuncture

Model

(b)

FIGURE 7: Acupuncture made a positive regulation of GSK-3 $\beta$, PP2A, and G/P. GSK-3 $\beta$ expression and G/P significantly increased in MCAO groups but decreased in acupuncture group $(P<0.05)$. PP2A expression showed a reverse tendency. The expression of GSK-3 $\beta$ and PP2A had a temporal profile. But acupuncture had a positive regulation for GSK-3 $\beta$, PP2A expression, and G/P $(P<0.05)$.

as response to neuron injury [36, 37], therefore immunopositive cells in penumbra may migrate from the specific neurogenesis regions and differentiate to neurons or glial cells for subsequent functional recovery. As to nestin, acupuncture showed a strong effect on its mRNA expression. Nestin expression in brain was obviously elevated by ischemia infarction, but different tendency were found in cortex, hippocampus and striatum. High expression of nestin mRNA in cortex was coincident with some previous reports which presented in some neurons or neurogliocyte localized in boundary zone of infarction area and showed a temporal profile $[34,38,39]$. We consider these temporal neurogenesis 
TABLE 2: The top 10 enriched pathways of the differentially expressed genes.

\begin{tabular}{|c|c|c|c|}
\hline KEGG ID & KEGG pathways & $P$ value & Gene name \\
\hline 4740 & Olfactory transduction & 0 & $\begin{array}{l}\text { Olr841;Olr847;Olr845;Olr853;Olr852;Olr851;Olr850;Olr848;Olr } \\
\text { 1006;Olr1007;Olr1660;Olr878;Olr1057;Olr1012;Olr1658;Olr936; } \\
\text { Olr950;Olr943;Olr947;Olr951;Olr1002... }\end{array}$ \\
\hline 471 & D-Glutamine and D-glutamate metabolism & 0 & Gls;Glud1;Gls2 \\
\hline 4080 & Neuroactive ligand-receptor interaction & $3.46 E-12$ & $\begin{array}{l}\text { Lpar2;Prss1;Prl;Prlr;Ptgfr;Tshb;Adrb3;Glra1;Cckbr;Mc5r;Chrng; } \\
\text { Prss3;Hrh1;Htrla;Lepr;Glra2;Gh1;Ednra;Drd2;Drd1a;Lpar3;Npy } \\
\text { 2r;LOC286960;Grik4;Grin1;Grm3;Grm1;Grin2c. .. }\end{array}$ \\
\hline 4610 & Complement and coagulation cascades & $4.52 E-06$ & $\begin{array}{l}\text { Plat;Serpina1;Serpine1;Clqc;Mbl1;Serpinc1;F5;Fgg;Fgb;Pros1;F } \\
\text { ga;Thbd;Kng1;F9;Bdkrb1;Plaur;C1s;Vwf;C3ar1;C7;C9;C5;Cr1l; } \\
\text { Cfd;Cfh;C8g;Serpina5;C1r;Serping1;F7;Cd55;Masp1;F13a1;Cr2; } \\
\text { Serpind1;Kng2;Klkb1;Masp2;F8;A2m;F13b;C4bpa;C4a;C6;C2;C } \\
\text { 3;Cpb2;C5ar1;F10;Mbl2;Clqa;F2r;Plau;F3;Proc;Bdkrb2;C1qb;C } \\
\text { 8a }\end{array}$ \\
\hline 5150 & Staphylococcus aureus infection & $4.68 E-06$ & $\begin{array}{l}\text { Selp;C1qc;Mbl1;Fcgr3a;Fgg;RT1-Ha;Fcgr2a;C1s;Dsg1b;Krt10;C } \\
\text { 3ar1;C5;Fcar;Fcgrla;Cfd;Ptafr;Cfh;C1r;Masp1;LOC688090;Itga } \\
\text { m;Masp2;Fcgr2b;Fpr21;Itgb2;RT1-Ba;RT1-Bb;RT1-DMa;RT1-D } \\
\text { Mb;RT1-Db1;RT1-Da;C4a;C2;C3;C5ar1;Selplg;Mbl2;C1qa;Icam } \\
\text { 1;LOC498276;Il10;C1qb;Itgal }\end{array}$ \\
\hline 4640 & Hematopoietic cell lineage & $6.65 E-06$ & $\begin{array}{l}\text { Il7;Mme;Il2ra;Cd3d;Il6;Il5;Il6r;Illa;Il1b;Il3;Il9r;Epo;Epor;Itga6; } \\
\text { Anpep;Cd36;Cd37;Il3ra;Cd9;Cd8a;Cd8b;Cd4;Ms4a1;Tnf;Il7r;Csf } \\
\text { 2;Gp1bb;Cd19;Csf1;RGD1565355;Il5ra;Fcgr1a;Gp9;Flt3;Itga5;K } \\
\text { itlg;Itga2;Tpo;Illr2;Cd3e;Csf1r;Kit;Cd55;Csf2ra;Cd14;Cr2;Cd3g; } \\
\text { Il4ra;Itgam;Cd24;Cd7;Cd1d1;Itgb3;LOC687856;RT1-Db1;RT1-D } \\
\text { a;Thpo;Csf3r;Cd44;Dntt;Csf3;Il4;Ill1;Fcer2;Gp5 }\end{array}$ \\
\hline 100 & Biosynthesis of steroids & $6.69 E-05$ & $\begin{array}{l}\text { Cyp27b1;LOC691221;Soat1;Tm7sf2;Cyp2r1;Nsdhl;Sc5dl;Ebp;D } \\
\text { hcr7;Hsd17b7;Lipa;Fdft1;Cel;Sqle;Cyp51;Msmo1;Soat2;Dhcr24 }\end{array}$ \\
\hline 4742 & Taste transduction & $7.05 E-05$ & $\begin{array}{l}\text { Scnn1g;Scnn1b;Itpr3;Kcnb1;Gnat3;Gnb1;Grm4;Gng13;Gnas;Tas } \\
\text { 2r137;Tas2r138;Tas2r126;Trpm5;Tas2r108;Tas2r102;Tas2r114;Ta } \\
\text { s2r121;Tas2r118;Tas2r107;Tas2r13;Tas2r105;Tas2r119;Tas2r144; } \\
\text { Gng3;Tas2r140;Adcy4;Tas2r143;Tas2r135;Prkx;Plcb2;Tas1r3;Tas } \\
\text { 2r134;Tas2r123;Tas1r1;Scnn1a;Tas2r120;Adcy8;Cacnalb;Tas2r1 } \\
\text { 39;Adcy6;Tas2r136;Asic2 }\end{array}$ \\
\hline 5323 & Rheumatoid arthritis & $7.35 E-05$ & $\begin{array}{l}\text { Cxcl12;Tnfsf13b;Cd28;Ctsl1;Ifng;Acp5;Il6;Ccl12;Illa;Il1b;Jun;L } \\
\text { tb;Vegfa;Ctsk;Ccl5;Il18;Tcirg1;Atp6vla;Atp6ap1;RT1-Ha;Tnf;At } \\
\text { p6v0d1;Atp6vle2;Tlr2;Atp6v1g2;Atp6vlf;Csf2;Tnfsf11;Atp6vlb } \\
\text { 2;Csf1;Cd86;Flt1;Il23a;Mmp1a;Cxc15;Atp6v0c;Atp6v1b1;Atp6v } \\
\text { lh;LOC688090;Atp6vlg3;Fos;Atp6v0a4;Ctla4;Ccl20;Tgfb1;Atp } \\
\text { 6v0e1;Itgb2;RT1-Ba;RT1-Bb;RT1-DMa;RT1-DMb;RT1-Db1;RT } \\
\text { 1-Da;Atp6v0e2;Tlr4;Cd80;Icam1;Tnfsf13;Angpt1;Ccl3;Il11;Mm } \\
\text { p3;Tnfrsf11a;Itgal;Ill7a }\end{array}$ \\
\hline 4060 & Cytokine-cytokine receptor interaction & 0.000178 & $\begin{array}{l}\text { Prl;Prlr;Cxcl12;Il22;Tnfsf13b;Il7;Met;Pdgfrb;Cntf;Il2ra;Ifng;Il2r } \\
\text { b;Il28ra;Il22ra1;Il6;Il5;Ccl12;Il6r;Illa;Il1b;Il3;Ifnb1;I19r;Ccl28; } \\
\text {.. }\end{array}$ \\
\hline
\end{tabular}

phenomenons accompanied by alleviation of neural functional damage in model group and no-acupuncture group as "self-repairing". It means focal ischemia damage could be in spontaneous remission to some extent even without any intervention. Among three brain regions, striatum got the highest expression immediately after MCAO, followed by cortex and hippocampus. However, "self-repairing" capacity in MCAO rats' brain reversed such tendency as mentioned above 3 days after MCAO: Nestin expression in cortex and hippocampus continued to increase while it decreased in striatum. Robustly increasing nestin expression in cortex demonstrated that acupuncture could enhance the "self-repairing" capacity, (while nestin expression slightly increased in hippocampus and decreased in striatum). Thus, we speculate that acupuncture stimulation on DU26, who is the key acupoint of Xing Nao Kai Qiao acupuncture therapy prescription, may influence on facilitating neural stem cells migration and enhancing "self-repairing" capacity in MCAO rats.

Intriguingly, compared with model group, improvement of neural functional damage found in no-acupuncture group and acupuncture group were not accompanied by reduction in infarction volume, which suggests that neural functional improvement did not correspond to significant decrease of infarction volume. That may be because only little part of 
newborn stem cells survived which is too few to reach morphological changing [8], but survived newborn stem cells had participated in neural circuit to function.

Given the results mentioned above, we speculate that acupuncture may achieve neuroprotection by enhancing selfrepairing capacity which increases cerebral blood flow to create an appropriate microenvironment for subsequent proliferation and migration of neural stem cell towards the ischemic boundary region to replenish damaged neuron. However, it is a long process for a neural stem cell to develop into a fully functional neuron. It includes neural stem cell generation, progenitor cell amplifying, differentiation, migration, synapses and neural circuit formation in a preexisting neural system. To determine the exact role of acupuncture in alleviation of neural damage in $\mathrm{MCAO}$, microarray analysis was employed. Microarray analysis is helpful to identify groups of genes distinctively changed among groups. The microarray results revealed that many genes and pathways were involved which were mainly relevant to phosphorylation, immunologic tolerance, protein regulation and cell proliferation functions by GO and pathway analysis. From the result of microarray assay, we found out that acupuncture treatment partly took effect by enhancing "self-repairing" capacity but was also mediated by gene transcription regulation involving many biological processes. All of them have potential for exploring ischemia treatment strategy.

To verify the results of microarray, measurement of protein GSK-3 $\beta$ and PP2A which play a crucial role in the above biological process was conducted. Glycogen synthase kinase$3 \beta$ (GSK-3 $\beta$ ) and protein phosphatase $2 \mathrm{~A}$ (PP2A) are important regulators of protein phosphorylation in many pathway. Protein phosphorylation known to a key regulation mechanism of cellular signal transduction is activator for neurons function as protein regulation and proliferation by $\beta$-catenin or MARK pathway. GSK-3 $\beta$, initially considered as a regulator of glycogen metabolism highly expressed in CNS [40], now is important in controlling protein synthesis, cell proliferation, differentiation and apoptosis [41]. PP2A negatively regulate GSK-3 $\beta$ and signal transduction of subsequent pathway by dephosphorylation. Mediation balance of GSK$3 \beta$ (upregulate) and PP2A (downregulate) expression have been associated with regulation of CNS neurons. Here, we found out that high expression of GSK- $3 \beta$ and suppression of PP2A were evoked by ischemia damage but regulated by acupuncture treatment in vivo, which suggests that mechanism of acupuncture neuroprotection may be associated with regulation GSK-3 $\beta$ and PP2A expression. However, further study is needed to determine which part is exactly participating in regulation of endogenous neurogenesis and genes expression changing post-ischemia damage.

Taken all the results together, we found out that the mechanism of acupuncture therapeutic effect on focal ischemia infarction is extremely complex. It is not only regulated by one single or two elements but involved multiple targets to achieve neural protective effect. The concept is consistent with old system theory of Traditional Chinese Medicine which is distinct from reductionism of modern medicine. The integrative system theory holds that human has a balance system that consists of many organs, and also a part of society and environment. Thus, multiple strategies are needed to diseases treatment. The Xing nao kai qiao acupuncture prescription consists of DU26, P6, SP6, LI11 and B40, established by Dr. Xuemin Shi for stroke treatment in 1970's. He holds that the main pathogenesis of stroke is "blockage of brain lead to spirit away". The spirit is the impetus of life activities in traditional medicine theory who is $q i$ in essence governed by brain. It governs human life activities through $q i$ from brain combine qi from kidney together then to trigger other organs' $q i$. Thus, the treatment principle of stroke should unblock the blockage in brain and promote circulation of $q i$ in body. Here, we selected DU26 (locating at the upper one-third and lower one-third of the cleft lip midline) in the prescription for it belong to the governor meridian that is traveling through brain and govern qi of body. It also follows the nearby acupuncture prescription principle for stroke locating on brain. And, modern anatomical study show that its skin innervates by trigeminal and deeper muscle innervates by facial nerve. So stimulating DU26 means stimulate trigeminal and facial nerve directly and the nerve impulse conduct to nucleus nervi facials and trigeminal nerve nuclei in brainstem. Confined by methodology, we have to study one acupoint each time, so we stimulated DU26 to determine the underlying mechanism of acupuncture on ischemia damage. Thus, more researches are needed.

In conclusion, the present study indicated that the underlying mechanism of acupuncture treatment on neural functional damage caused by focal ischemia injury was a multiple action which may be associated with improved cerebral blood supply, neurogenesis and regulation of transcription or GSK$3 \beta$ and $\mathrm{PP} 2 \mathrm{~A}$ expression in MCAO rats. Acupuncture is a potential therapeutic strategy for ischemia stroke.

\section{Conflict of Interests}

The authors declare that there is no conflict of interests regarding the publication of this paper.

\section{Authors' Contribution}

Ding Luo and Xiaonong Fan equally contributed to this work as joint first authors.

\section{Acknowledgment}

This study is supported by the national science and technology support program of the twelfth Five-Year Plan (No: 2013BAI13B03), the Special Program For Key Fundamental Research Development Project of China (No: 2010CB530506) and the Natural Science Foundation of Tianjin city (No: 10JCBJC2700).

\section{References}

[1] M. Liu, B. Wu, W. Wang, L. Lee, S. Zhang, and L. Kong, "Stroke in China: epidemiology, prevention, and management strategies," The Lancet Neurology, vol. 6, no. 5, pp. 456-464, 2007. 
[2] G. Xu, M. Ma, X. Liu, and G. J. Hankey, "Is there a stroke belt in China and why?" Stroke, vol. 44, no. 7, pp. 1775-1783, 2013.

[3] J. Altman, "Autoradiographic and histological studies of postnatal neurogenesis. IV. Cell proliferation and migration in the anterior forebrain, with special reference to persisting neurogenesis in the olfactory bulb," Journal of Comparative Neurology, vol. 137, no. 4, pp. 433-457, 1969.

[4] M. S. Kaplan and J. W. Hinds, "Neurogenesis in the adult rat: electron microscopic analysis of light radioautographs," Science, vol. 197, no. 4308, pp. 1092-1094, 1977.

[5] F. H. Gage, G. Kempermann, T. D. Palmer, D. A. Peterson, and J. Ray, "Multipotent progenitor cells in the adult dentate gyrus," Journal of Neurobiology, vol. 36, no. 2, pp. 249-266, 1998.

[6] H. van Praag, A. F. Schinder, B. R. Christle, N. Toni, T. D. Palmer, and F. H. Gage, "Functional neurogenesis in the adult hippocampus," Nature, vol. 415, no. 6875, pp. 1030-1034, 2002.

[7] R. Zhang, Z. Zhang, L. Wang et al., "Activated neural stem cells contribute to stroke-induced neurogenesis and neuroblast migration toward the infarct boundary in adult rats," Journal of Cerebral Blood Flow and Metabolism, vol. 24, no. 4, pp. 441-448, 2004.

[8] A. Arvidsson, T. Collin, D. Kirik, Z. Kokaia, and O. Lindvall, "Neuronal replacement from endogenous precursors in the adult brain after stroke," Nature Medicine, vol. 8, no. 9, pp. 963970, 2002.

[9] D. Lu, A. Mahmood, C. Qu, A. Goussev, T. Schallert, and M. Chopp, "Erythropoietin enhances neurogenesis and restores spatial memory in rats after traumatic brain injury," Journal of Neurotrauma, vol. 22, no. 9, pp. 1011-1017, 2005.

[10] S. S. Magavi, B. R. Leavitt, and J. D. Macklis, "Induction of neurogenesis in the neocertex of adult mice," Nature, vol. 405, no. 6789, pp. 951-955, 2000.

[11] M. J. During and L. Cao, "VEGF, a mediator of the effect of experience on hippocampal neurogenesis," Current Alzheimer Research, vol. 3, no. 1, pp. 29-33, 2006.

[12] P.-F. Shen, L. Kong, L.-W. Ni et al., "Acupuncture intervention in ischemic stroke: a randomized controlled prospective study," The American Journal of Chinese Medicine, vol. 40, no. 4, pp. 685-693, 2012.

[13] X. L. Li, N. X. Fan, H. Z. Meng, M. X. Shi, D. Luo, and N. Y. Zhang, "Specific neuroprotective effects of manual stimulation of real acupoints versus non-acupoints in rats after middle cerebral artery occlusion," African Journal of Traditional, Complementary and Alternative Medicines, vol. 10, no. 4, pp. 186-195, 2013.

[14] Y. N. Zhang, S. Yang, X. N. Fan et al., "Orthogonal design to sift the optimal parameter of Neiguan acupuncture for cerebral infarction," Neural Regeneration Research, vol. 8, no. 28, pp. 2641-2648, 2013.

[15] E. Z. Longa, P. R. Weinstein, S. Carlson, and R. Cummins, "Reversible middle cerebral artery occlusion without craniectomy in rats," Stroke, vol. 20, no. 1, pp. 84-91, 1989.

[16] J. M. Wojtowicz and N. Kee, "BrdU assay for neurogenesis in rodents," Nature Protocols, vol. 1, no. 3, pp. 1399-1405, 2006.

[17] S. Zausinger, E. Hungerhuber, A. Baethmann, H. Reulen, and R. Schmid-Elsaesser, "Neurological impairment in rats after transient middle cerebral artery occlusion: a comparative study under various treatment paradigms," Brain Research, vol. 863, no. 1-2, pp. 94-105, 2000.

[18] R. A. Swanson, M. T. Morton, G. Tsao-Wu, R. A. Savalos, C. Davidson, and F. R. Sharp, "A semiautomated method for measuring brain infarct volume," Journal of Cerebral Blood Flow and Metabolism, vol. 10, no. 2, pp. 290-293, 1990.

[19] M. E. O'Donnell, L. Tran, T. I. Lam, X. B. Liu, and S. E. Anderson, "Bumetanide inhibition of the blood-brain barrier $\mathrm{Na}-\mathrm{K}-\mathrm{Cl}$ cotransporter reduces edema formation in the rat middle cerebral artery occlusion model of stroke," Journal of Cerebral Blood Flow and Metabolism, vol. 24, no. 9, pp. 10461056, 2004

[20] K. J. Livak and T. D. Schmittgen, "Analysis of relative gene expression data using real-time quantitative PCR and the $2^{-\Delta \Delta C_{T}}$ method," Methods, vol. 25, no. 4, pp. 402-408, 2001.

[21] D. N. Abrous, M. Koehl, and M. Le Moal, "Adult neurogenesis: from precursors to network and physiology," Physiological Reviews, vol. 85, no. 2, pp. 523-569, 2005.

[22] J. Boltze, C. Kleinschnitz, K. G. Reymann et al., "Neurovascular pathophysiology in cerebral ischemia, dementia and the ageing brain-current trends in basic, translational and clinical research," Experimental and Translational Stroke Medicine, vol. 4, article 14, 2012.

[23] N. Tajiri, Y. Kaneko, K. Shinozuka et al., "Stem cell recruitment of newly formed host cells via a successful seduction? Filling the gap between neurogenic niche and injured brain site," PLoS ONE, vol. 8, no. 9, Article ID e74857, 2013.

[24] G. J. Lee, C. S. Yin, S. K. Choi, J. S. Yang, H. Lee, and H. K. Park, "Acupuncture attenuates extracellular glutamate level in global ischemia model of rat," Neurological Research, vol. 32, supplement 1, pp. 79-83, 2010.

[25] J. H. Chung, E. Lee, M. Jang et al., "Acupuncture decreases ischemia-induced apoptosis and cell proliferation in dentate gyrus of gerbils," Neurological Research, vol. 29, supplement 1, pp. S23-S27, 2007.

[26] F. Zhou, J. Guo, J. Cheng, G. Wu, J. Sun, and Y. Xia, "Electroacupuncture and brain protection against cerebral ischemia: specific effects of acupoints," Evidence-Based Complementary and Alternative Medicine, vol. 2013, Article ID 804397, 14 pages, 2013.

[27] K. A. Kang, E. S. Shin, J. Hur et al., "Acupuncture attenuates neuronal cell death in middle cerebral artery occlusion model of focal ischemia," Neurological Research, vol. 32, supplement 1, pp. 84-87, 2010.

[28] H. Liu, X. Shen, H. Tang, J. Li, T. Xiang, and W. Yu, "Using MicroPET imaging in quantitative verification of the acupuncture effect in ischemia stroke treatment," Scientific Reports, vol. 3, article 1070, 2013.

[29] Y. F. Qian, X. Fan, Y. Li et al., "Effects of acupuncture at different acupoints on cerebral blood flow in cerebral ischemia model rats," Zhongguo Zhen Jiu, vol. 29, no. 3, pp. 213-216, 2009.

[30] Z. Wang, X. Ma, H. Liu et al., "Effects of eye-acupuncture therapy on the expression of AQP4 in brain tissue of rats with acute cerebral ischemia-reperfusion injury," Zhongguo Zhen Jiu, vol. 31, no. 8, pp. 727-731, 2011.

[31] X. N. Fan, S. Wang, Y. Li et al., "Study on optimum stimulation parameter for Shuigou (GV 26) in Xingnao Kaiqiao needling method," Zhongguo Zhen Jiu, vol. 28, no. 12, pp. 913-917, 2008.

[32] Y. Du, L. Shi, J. Li, J. Xiong, B. Li, and X. Fan, "Angiogenesis and improved cerebral blood flow in the ischemic boundary area were detected after electroacupuncture treatment to rats with ischemic stroke," Neurological Research, vol. 33, no. 1, pp. 101107, 2011.

[33] U. Lendahl, L. B. Zimmerman, and R. D. G. McKay, "CNS stem cells express a new class of intermediate filament protein," Cell, vol. 60 , no. 4 , pp. 585-595, 1990. 
[34] Y. Li and M. Chopp, "Temporal profile of nestin expression after focal cerebral ischemia in adult rat," Brain Research, vol. 838, no. 1-2, pp. 1-10, 1999.

[35] S. Hockfield and R. D. G. McKay, "Identification of major cell classes in the developing mammalian nervous system," The Journal of Neuroscience, vol. 5, no. 12, pp. 3310-3328, 1985.

[36] O. Lindvall and Z. Kokaia, "Recovery and rehabilitation in stroke: stem cells," Stroke, vol. 35, no. 11, supplement 1, pp. 26912694, 2004.

[37] P. S. Eriksson, E. Perfilieva, T. Björk-Eriksson et al., "Neurogenesis in the adult human hippocampus," Nature Medicine, vol. 4, no. 11, pp. 1313-1317, 1998.

[38] W. Guo, N. E. Patzlaff, E. M. Jobe, and X. Zhao, "Isolation of multipotent neural stem or progenitor cells from both the dentate gyrus and subventricular zone of a single adult mouse," Nature Protocols, vol. 7, no. 11, pp. 2005-2012, 2012.

[39] H. Y. Shin, J. H. Kim, J.H. Phi et al., "Endogenous neurogenesis and neovascularization in the neocortex of the rat after focal cerebral ischemia," Journal of Neuroscience Research, vol. 86, no. 2, pp. 356-367, 2008.

[40] J. R. Woodgett, "Molecular cloning and expression of glycogen synthase kinase-3/factor A," EMBO Journal, vol. 9, no. 8, pp. 2431-2438, 1990.

[41] C. F. Lin, C. Chen, C. Chiang, M. Jan, W. Huang, and Y. Lin, "GSK-3 $\beta$ acts downstream of PP2A and the PI 3-kinase-Akt pathway, and upstream of caspase- 2 in ceramide-induced mitochondrial apoptosis," Journal of Cell Science, vol. 120, part 16, pp. 2935-2943, 2007. 


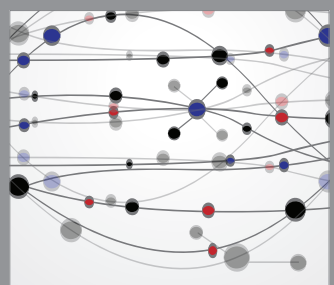

The Scientific World Journal
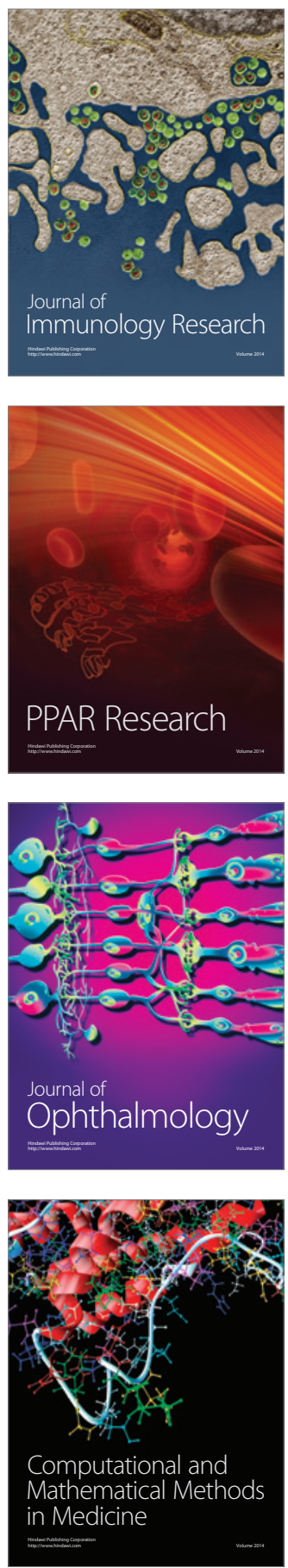

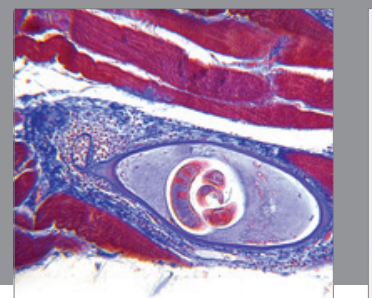

Gastroenterology

Research and Practice
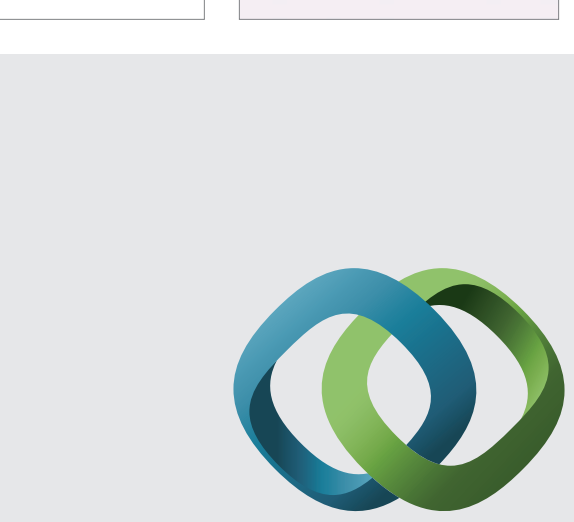

\section{Hindawi}

Submit your manuscripts at

http://www.hindawi.com
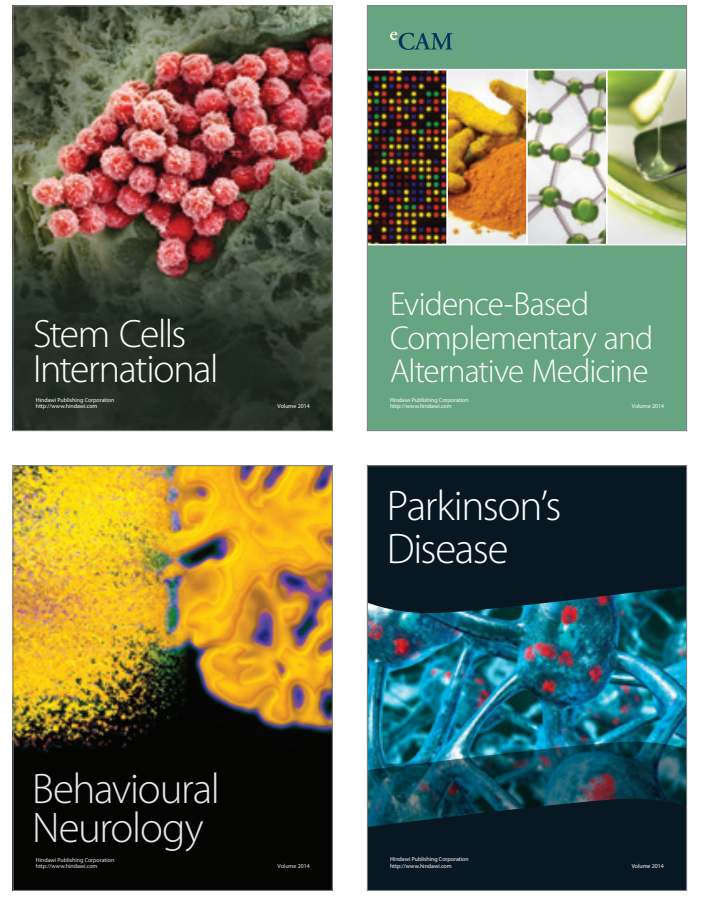
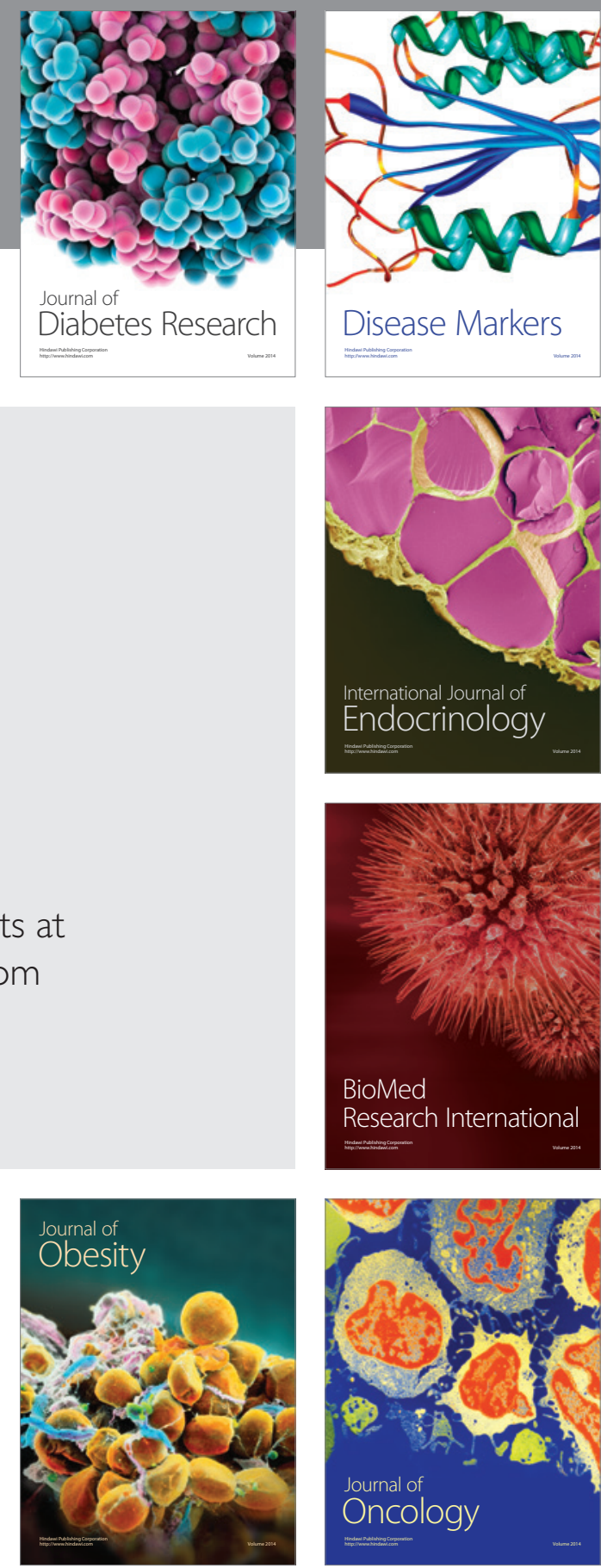

Disease Markers
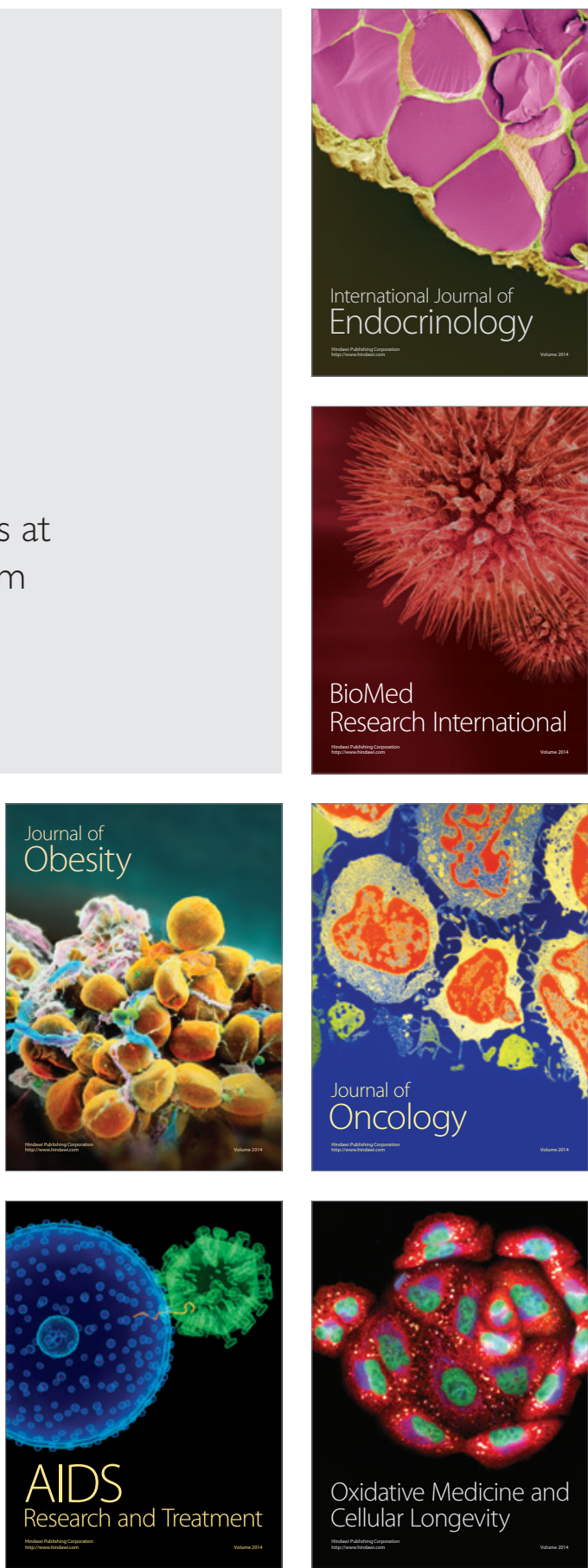